\title{
Using a Formal Declarative Language for Specifying Requirements Modelled in CIMOSA
}

\author{
Eric Dubois, Michaël Petit \\ Facultés Universitaires de Namur, Institut d'Informatique \\ Rue Grandgagnage 21, B-5000 Namur (Belgium) \\ \{edu, mpe\}@ info.fundp.ac.be
}

\begin{abstract}
Requirements Engineering is more and more considered as a central phase in the development and implementation of computer systems. Within the context of CIM, the CIMOSA project proposes a set of models based on adequate concepts for expressing requirements. In this paper, we suggest how these models can be supported by the use of a fully formal requirements specification language called ALBERT and based on an agent-oriented real-time temporal logic framework.
\end{abstract}

\section{Keywords}

Requirements Engineering, CIMOSA, ALBERT, real-time temporal logic, agent-oriented framework.

\section{INTRODUCTION}

It is now widely recognised that the implementation and the maintenance of successful and adequate CIM infrastructures can only be achieved through the adoption of a rigourous development process made of a number of well-defined activities covering the whole lifecycle of a CIM infrastructure.

Among these activities, requirements analysis (or requirements engineering (RE)) is an activity which appears as crucial since it is in charge of eliciting and capturing customers wishes and goals for the CIM infrastructure to be settled in an enterprise. From recent research trends related to the RE activity, we can learn that a key issue relies on the use of an adequate language for modelling the requirements expressed by customers. Two specific qualities are expected from such a language:

- On the one hand, the language should be expressive enough so that customers requirements can be modelled in a natural way, without the introduction of any overspecification. To ensure this traceability property, a suitable requirements language will be based on an ontology 
of concepts large enough so that it permits a straightforward modelling of a large variety of requirements belonging to the considered application domain.

- On the other hand, the language should be a formal language in order to support powerful verification and validation checks, i.e. a language equipped with an adequate mathematical/logical semantics made of (i) rules of interpretation which will guarantee the absence of ambiguities in a requirements document and (ii) rules of deduction which will permit to reason on a specification document in order to discover potential incompletenesses and/or inconsistencies.

Within the CIM context, languages have been proposed for the purpose of modelling requirements. Examples include, e.g., SADT (Ross, 1977), IDEF-0 (CAM.I., 1980), ... However, such languages are not really formal languages since they are mainly based on a set of 'boxes and arrows' notations with only a poor underlying semantics. Another problem is that such languages have been originally designed for the purpose of modelling requirements inherent to business information systems and, thereby, it is not proved that their underlying ontology of concepts is rich enough for capturing the whole complexity of a CIM enterprise modelling. Among some initiatives at the level of the identification of an adequate ontology of concepts, one may quote the CIMOSA project (ESPRIT Consortium AMICE, 1993), (Vernadat, 1993) which will be heavily referred throughout the rest of this paper.

At the level of formality, there are formal languages proposed for specifying CIM applications. Some examples include (Bastide, 1991), (Jaulent, 1990), (Zerhouni, 1990) and are based on Petri-Nets and/or on an operational semantics. However, such languages are basically design specification languages and not requirements specification languages. This means that there are more adequate for expressing the solution to a problem rather than to model the problem itself. In most cases, such languages encourage a procedural style of specification while a more declarative (logical) style is required at the RE level.

Some recent examples of formal requirements specification languages include RML (Greenspan, 1986), GIST (Feather, 1987), MAL (Finkelstein, 1987) and ERAE (Dubois, 1991). In the specific CIM context, as examples, one may quote the on-going researches of Bussler (Bussler, 1993) related to the use of workflows and the work of Fox (Fox, 1994) relying on a specific logical framework. In this paper, we propose to evaluate the ALBERT language, a language that has been designed recently with the purpose of modelling requirements inherent to distributed real-time safety-critical systems. In order to proceed to this evaluation, the expressiveness of ALBERT will be judged through its capacity of modelling concepts presented in the CIMOSA RE framework (viz. domains, processes, inputs and outputs, resources, etc).

All along this paper, we will illustrate the use of ALBERT by considering a small case study, originally introduced in (Lutherer, 1994) for the purpose of illustrating the application of the CIMOSA concepts at the requirements and design levels.

The example concerns the automation of a concrete batching and mixing plant. The goal of the installation is to produce several types of concrete corresponding to the customer's demand. For that purpose, the plant is composed of:

- several bins to store the cements, the aggregates and the water;

- a mixer, for the preparation of the concrete;

- two scales, to weight the ingredients;

- two conveyor belts, to carry the ingredients from the bins to the mixer. 


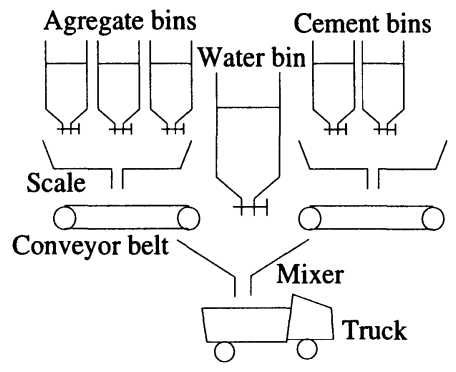

Figure 1 Schema of the plant.

The objective of the system is to produce automatically the concrete corresponding in composition, quantity and delay to the customer order. The system is depicted on Figure 1.

In Sect.2, an in-depth presentation of the ALBERT language is provided together with its illustration through fragments of the case study presented above. In Sect. 3, we carefully review the ontology of concepts made available in CIMOSA for the modelling purposes and we compare each of them with an equivalent construct available in ALBERT. Finally, Sect. 4 concludes by providing a brief overview of our recent researches in the CIM context.

\section{THE ALBERT LANGUAGE}

In this paper, we do not provide an in-depth presentation of the ALBERT language (such presentation can be found in (Dubois, 1994b)). Our aim is just to illustrate some basic features of the language through the handling of the concrete plant case study.

Basically, ALBERT is based on a variant of real-time temporal logic, a mathematical language particularly suited for describing histories (i.e. sequences of states) and expressing performances constraints (like, e.g. "this property holds for at least 3 minutes"). This logic is itself an extension of multi-sorted first order logic, still based on the concepts of variables, predicates and functions. The language introduces mainly three extensions:

1. the introduction of actions. Actions are associated with changes that may alter states in histories. Using actions in ALBERT makes possible to overcome the well-known frame problem, a typical problem resulting from the use of a declarative specification language;

2. the introduction of agents. An agent, which can be seen as a specialization of the object concept, is characterized through (i) its internal state recording the knowledge maintained by the agent and the "visibility" that it offers to the other agents of the environment, (ii) its perception of what is happening in its environment and (iii) its responsibility with respect to actions having some effects on its state or on states of other agents.

3. the identification of typical patterns of constraints. The use of a logical formal language may be compared to the use of an assembly programming language. A set of basic constructs are available in the language but there is a lack of support for the analyst in writing complex and consistent statements. To overcome this problem, a number of typical patterns of 


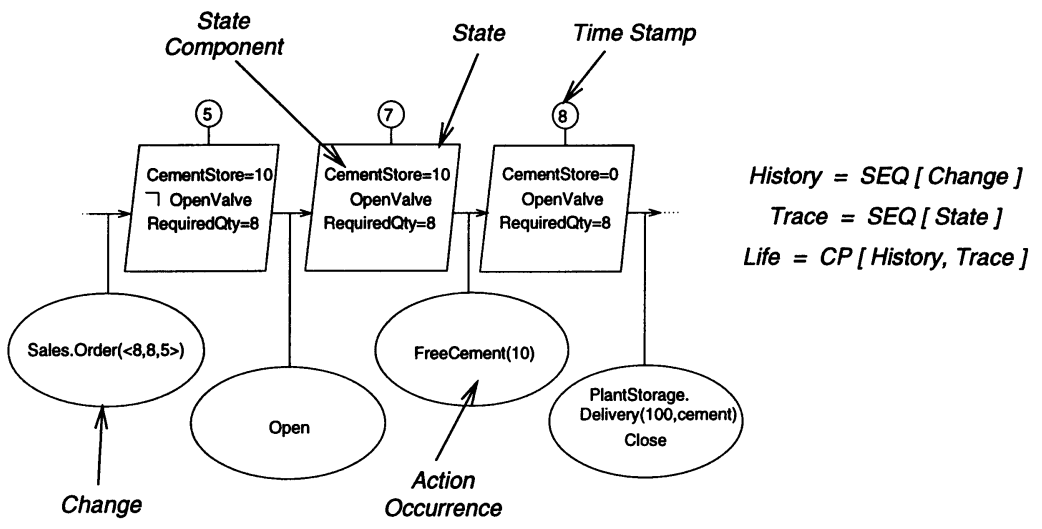

Figure 2 A graphical representation of a possible life of a CementBin agent (excerpt).

formulas have been predefined in ALBERT (in the past, a similar need for typical patterns was identified in RML (Greenspan, 1986)).

Using the language involves two activities: (i) writing declarations introducing the vocabulary of the considered application and (ii) expressing constraints, i.e. logical statements which identify possible behaviours of the different agents and exclude unwanted ones. A graphical syntax (with a textual counterpart) is used to introduce declarations and to express some static properties. The expression of the other constraints is purely textual.

Before to present the language constructs in Sect.2.2 and 2.3, we first provide a brief insight of the mathematical model underlying an ALBERT specification.

\subsection{Models of a Specification}

The purpose of our requirements language is to define admissible behaviours of the system to be developed. A specification language is best characterized by the structure of models it is meant to describe.

In order to master their complexity, models of a specification are derived at two levels:

- at the agent level: a set of possible behaviours is associated with each agent without any regard to the behaviour of the other agents;

- at the society (group of agents) level: interactions between agents are taken into account and lead to additional restrictions on each individual agent behaviour.

The specification describes an agent by defining a set of possible lives modelling all its possible behaviours. A life is an (in)finite alternate sequence of changes and states; each state is labelled by a time value which increases all along the life. Figure 2 illustrates, in a graphical way, the concept of life by giving an excerpt of a possible life of the CementBin agent.

The term "history" refers to the sequence of changes which occur in a possible life of the agent. A change is composed of several occurrences of simultaneous actions (the absence of action is 


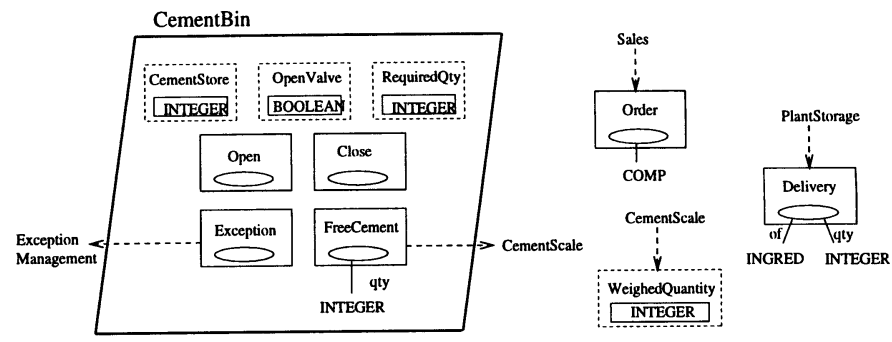

Figure 3 Declarations of the CementBin Agent.

also considered as a change). The term "trace" refers to a sequence of states being part of a possible life of the agent. A state is structured according to the information handled in the considered application in terms of state components. Notice that the value of a state at a given time in a certain life can always be derived from the initial state and the sub-history containing the changes occurred so far.

\subsection{Declarations}

\section{Declaration of Agents}

The declaration part of an agent consists in the description of its states structure and the list of the actions its history can be made of. Importation and exportation links between agents are also graphically described.

Since agents are considered as specialized objects, our modelling of a state structure is largely inspired by recent results in O-O conceptual modelling (see, e.g., OBLOG (Sernadas, 1989) and O* (Brunet, 1991)).

Agents include a key mechanism that allows the identification of the different instances. A type is automatically associated to each class of agent. For instance, each CementBin agent has an identifier of type CEMENTBIN.

The state is defined by its components which can be individuals or populations. Usually populations are sets of individuals but they can also be structured in sequences or tables. Elements of components are typed using (i) predefined elementary data types (like, STRING, BOOLEAN, IN$T E G E R, \ldots$, (ii) user-defined elementary types (for which no structure is given), (iii) user-defined constructed types built using predefined type constructors like,e.g. Cartesian product, sequence, union, enumerated type, etc (e.g. INGRED, in our example, is defined as an enumeration of three possible values, namely Cement, Aggregate and Water), or (iv) types corresponding to agent identifiers.

Figure 3 proposes the graphical diagram associated with the declaration of the CementBin agent. It can be read that:

- CementStore is an instance of type INTEGER indicating the current amount of cement in the bin;

- FreeCement is an action which may be issued by the CementBin. Actions can have arguments; for example, each occurrence of a FreeCement action has an instance of type INTEGER as argument which indicates the quantity of cement released by the bin when the valve is open. 


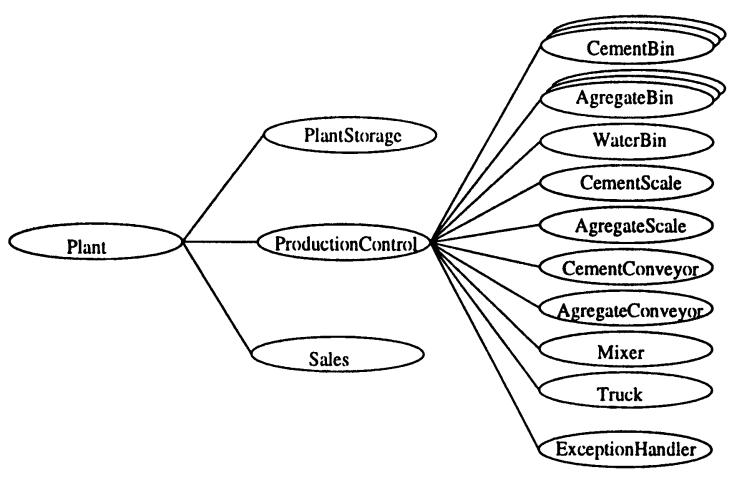

Figure 4 Declarations of the resources hierarchy of the concrete plant.

The diagrams also includes graphical notations used to express the visibility relationships linking the agent to the outside (Importation and Exportation mechanisms). Boxes without arrow denote information (state components or actions) which is not visible from the outside while boxes with arrow denote information which is exported to the outside. From the declaration of the CementBin agent, it can be read, for example, that the Exception Management department may have knowledge of Exception occurrences inside the bin and that the CementScale informs the bin of the currently WeighedQuantity.

Importation and Exportation are static properties; Perception and Information are their dynamic counterparts and provide the analyst with a finer way of controlling how agents can see information inside each other (perception and information constraints will be discussed in Sect. 2.3).

\section{Declaration of a Society}

Agents are grouped into societies. Societies themselves can be grouped together to form larger societies. In fact, a specification consists in a hierarchy of agents (a tree-like structure). Figure 4 shows the hierarchy associated with the concrete plant.

The existing hierarchy among agents is expressed in term of two combinators: cartesian product and set. In our specific case, e.g. the ProductionControl agent is an aggregate of one Mixer, several CementBins, one CementConveyor, one CementScale, a Truck, ...

\subsection{Constraints}

Constraints are used for pruning the (usually) infinite set of possible lives (histories) of an agent. Unlike usual design specification languages, the ALBERT semantics is not operational. A life must be extensively considered before it can be classified as possible or not, i.e. adding new states and changes at the end of a possible life does not necessarily result in a possible life.

Figure 5 introduces the specification associated with the behaviour of the CementBin agent and refers to the graphical declaration introduced in Figure 3. Formal constraints are expressed in terms of the different patterns available in the language. Rather than describing in details those patterns (which are extensively presented in (Dubois, 1994b), we will only refer to the informal comments written on the figure in order to exhibit the expressiveness and the non-operational style of an ALBERT specification.

Basically, properties are classified under 8 different headings grouped into two families: Local Constraints precise the responsibility of an agent with respect to changes that may alter its 
state of knowledge, and Cooperation Constraints specify how the agent interacts with the other agents of its environment. Finally, it should be also noted the use of Basic Constraints to define the initial values of the system.

\section{Local Constraints}

Local constraints are related to the internal behaviour of the agent. These constraints prescribe the sequence of actions that may/must happen through the whole life of the agent as well as the sequence of states which results from action occurrences. Local constraints are classified under four headings: State Behaviour, Effects of Actions, Causality and Capabilities.

\section{CementBin}

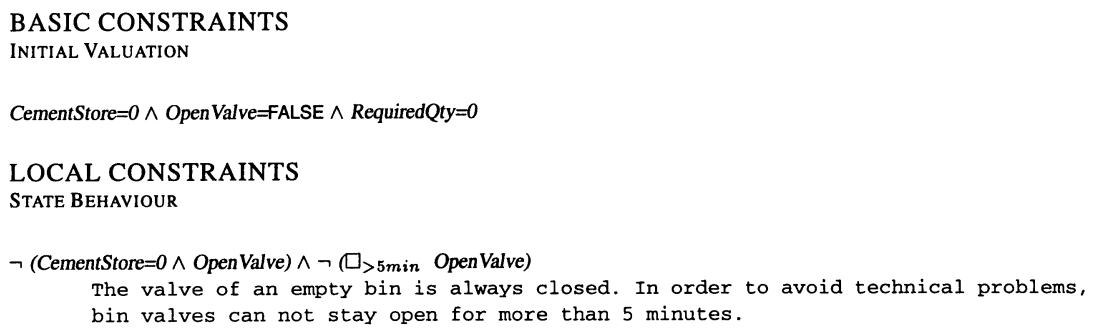




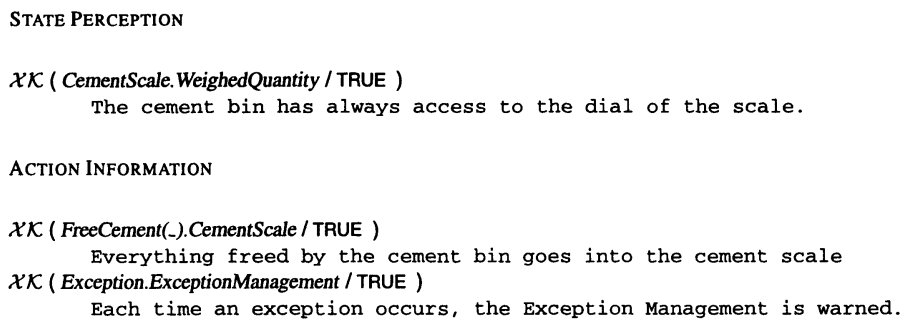

Figure 5 Constraints on the CementBin Agent.

Under the heading Effects of Actions are described the effects of the different actions happening. The effect of an action is expressed in terms of a property characterizing the state which follows the occurrence of an action (see examples in Figure 5). Under the heading Capability is described the responsibility of the agent with respect to the occurrences of its own actions. The default rule is that all actions are permitted whatever the situation but specific constraints can be added for making possible to express circumstances under which obligations and preventions are associated with actions occurrences (see for example the prevention constrain on the CementBin agent stating that no FreeCement action can occur when the bin valve is closed).

A specification of requirements only written with Effects of Actions and Capability constraints leads the analyst to adopt a rather operational style of specification where are described states transitions and conditions under which these transitions may/must occur (such a style of specification is advocated in, e.g., MAL (Finkelstein, 1987). Adopting this style may lead to the introduction of extra information in the agent state at the risk of over-specifications.

ALBERT provides more freedom to the analyst by letting him/her to adopt a more declarative style of specification. Under the Causality heading are described the causality relationships existing among action occurrences. In our case study, an example of causality exists among e.g. an Order request issued by the Sales department and a Open action under the control of the cement bin. It relies upon the necessity of having one unique occurrence of the Open action in response to each occurrence of the Order action. Finally, under the State Behaviour heading are described two kinds of constraints which must hold from the admissible history of an agent:

- static constraints are constraints which are true in all states (usually referred as invariants);

- dynamic constraints are constraints on the evolution of the state. They are expressed using temporal connectives. See for example the constrains on the CementBin agent.

\section{Cooperation Constraints}

Under the Action Perception and State Perception headings is described the responsibility of an agent with respect to the perception that it has to guarantee (i) for the actions happening in other agents and (ii) for state information made visible by other agents. In any case, using the 
appropriate pattern, the perception guaranteed by an agent may vary with time and depend on circumstances (see, the example of an Action Perception constraint in Figure 5 stating that $O r d e r$ requests issued by the Sales department are ignored if another order is currently being processed (RequiredQty $\neq 0$ ) or if not enough cement is present in the bin.

Under the Action Information and State Information headings is described the responsibility of an agent with respect to the visibility that it may offer to some other agents (i) for some of its performed actions and (ii) for parts of its state. See, in Figure 5, the example of an Action Information constraint in the specification of the CementBin agent stating that the FreeCement action is always showed to the CementScale agent. In any case, using the appropriate pattern, the visibility offered by an agent may vary with time, depend on circumstances and be restricted to some agents.

The use of perception and information constraints makes possible to describe various cooperation protocols (reliable or not) that may take place among agents. Again, ALBERT permits to express the different protocols at a high level of abstraction and without any regard to implementation details (like, e.g., the use of acknowledgment messages, messages storage buffers).

\section{FROM CIMOSA CONCEPTS TO ALBERT CONSTRUCTS}

The CIMOSA proposal (ESPRIT Consortium AMICE, 1993), (Vernadat, 1993) relies upon a framework made of different integrated models (with associated concepts and supporting formalisms) used for the purpose of covering the different phases (i.e. requirements definition, design and implementation) of the engineering activity of CIM systems.

In this section, we will concentrate on the relationship existing between ALBERT and CIMOSA at the requirements and design engineering levels. At the requirements level, CIMOSA concentrates on a logical view of the different functionalities to be performed in the CIM system without any regard to the resources required for their execution. These resources are taken into account at the design level where elementary functions identified in the functionalities are taken in charge by the resources.

Throughout this section, we will refer to the terminology used in CIMOSA and illustrate the mapping between CIMOSA and ALBERT through the case study already introduced and completely handled in (Lutherer, 1994).

\subsection{Requirements Definition}

In CIMOSA, the Requirements Definition Modelling Level concentrates on collecting the enduser needs and on structuring them in terms of a hierarchy of purely logical processes where:

- At the top level, processes correspond to the Domains Processes (DP) belonging to the Domains establishing the scope of the studied problem. DP's are further decomposed into Business Processes.

- At an intermediate level, processes correspond to Business Processes (BP). BP's identify the logical activities as well as the required sequencing of them needed for solving the manufacturing system problem. BP's can be themselves further decomposed into finer BP's and/or into Enterprise Activities. Within the context of our case study, Figure 6 shows an example of such decomposition. 


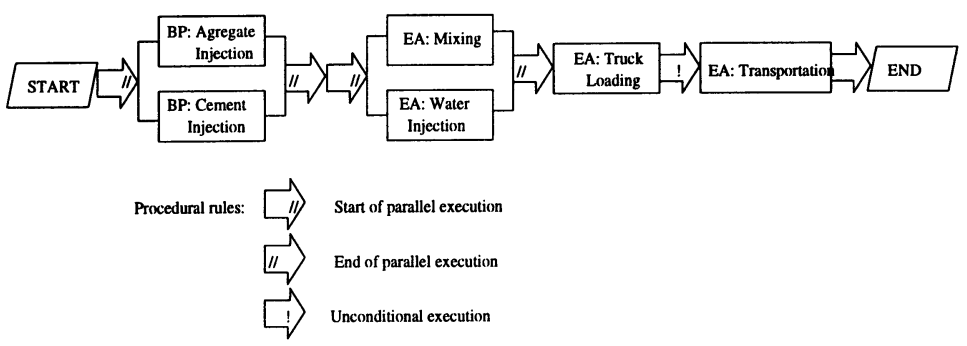

Figure 6 Decomposition and behaviour of the Production BP.

- At the lower level, processes correspond to Enterprise Activities (EA). EA's are terminal nodes in the hierarchy and are associated with the description of the ultimate functionalities (inputs/outputs transformations) guaranteeing the effectiveness of the solution to the manufacturing problem. Within the context of our case study, Figure 7 shows an example of such decomposition.

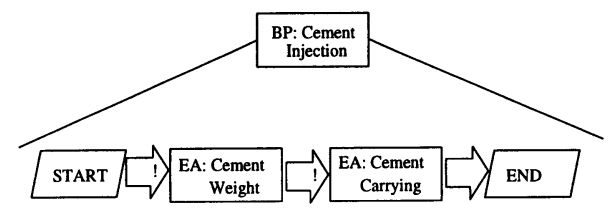

Figure 7 Decomposition and behaviour of the CementWeight BP.

\section{Processes and their behaviour}

Using ALBERT, it is straightforward to map the results of the Requirements Definition Modelling Level through the elaboration of an equivalent hierarchy where:

- societies (group of agents) are introduced at the higher and intermediate levels for representing DP's and BP's;

- agents are introduced at the lower levels for representing EA's.

Within the context of our case study, such hierarchy is presented on Figure 8.

In CIMOSA, at the Behaviour Analysis Level, Procedural Rules (PR) are used for defining the logical sequence of BP's and EA's inside a specific DP/BP (see Figure 6). Such rules have their counterpart in ALBERT where causalities (see Local Constraints in Sect. 2.3) can be used to express the unconditional execution, the conditional execution, the parallel execution and the rendezvous of different processes.

Besides the dynamical aspect, in CIMOSA, at the Operational Analysis level, ultimate elements introduced in the different functionalities are characterised by Inputs and Outputs. Inputs are of three types:

- function inputs are a set of object views (information) representing information and physical objects processed by the EA; 


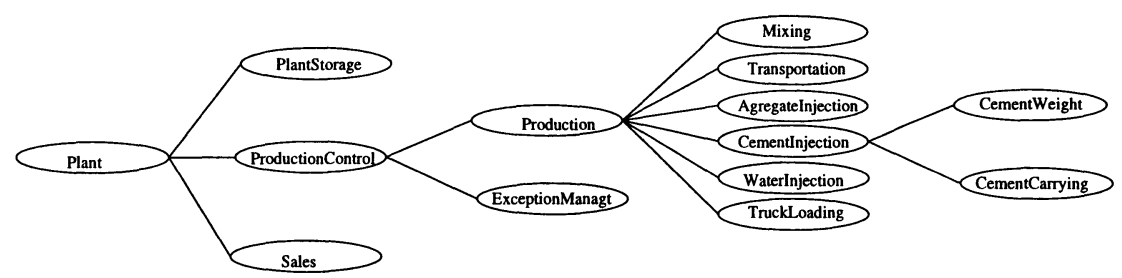

Figure 8 Declarations of the process hierarchy of the Production Control Domain.

- control inputs represent information used to control or to constrain the execution of the EA but which are not processed by the EA. A typical example is a NC-Programme;

- resource inputs are either the current resources used for executing the EA or a set of required capabilities of candidate resources. Required Capabilities may describe abilities of machines as well as of humans.

Outputs of an EA are also of three types:

- function outputs are, similarly to function inputs, a set of object views (information) representing information and physical objects produced by the EA;

- control outputs are a set of events generated by the EA and which may cause the triggering of a domain process;

- resource outputs report the information to be recorded about the usage of resources after execution of the EA (e.g. tool usage times, ...).
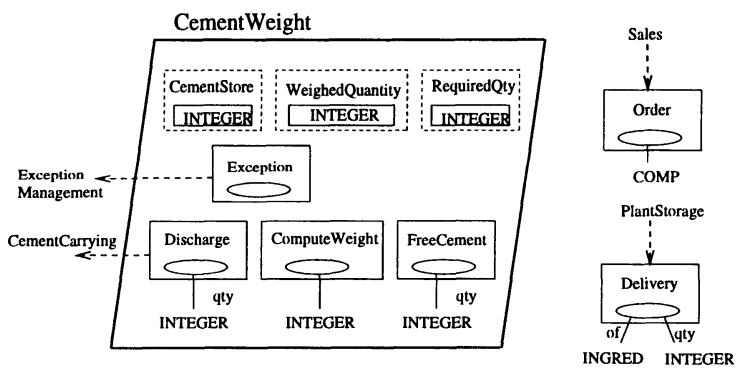

Figure 9 ALBERT declarations associated with the CementWeight EA.

In Figure 9, we provide the specification of the EA CementWeight where we have identified:

- RequiredQty as a control input recording the quantity of the cement to be weighed;

- CementStore as a function input attached with the stock of cement;

- Exception as a control output associated with the occurrence of an exception and the request issued to the Exception Management Domain Process to handle it;

- Weighed Quantity as a function output attached with the cement weighed before its delivery.

All the constraints attached with these different inputs/outputs are presented in Figure 10. 


\section{Information view}

In parallel with the specification of the different processes, CIMOSA also recommends to perform an Information Analysis based on an object oriented paradigm. The concepts used are $O b$ ject Views (perception of objects by users or applications), Information Elements (data items composing object views), Enterprise Objects (EOs) (entities of the enterprise on which views are defined), Object Abstraction Mechanisms (generalisation and aggregation between objects), Object Relationships (connecting pairs of objects), and Integrity Rules (constraints on information).

The information modelling in ALBERT is based on data types. Every information in a specification (be an action argument or a state component) is typed. Information Elements (IEs) correspond to simple (basic or predefined) data types. These are the basic elements (attributes) with are used to define more complex objects. Enterprise objects correspond to complex data types (defined by applying constructors -like Cartesian Product, Set, Sequence, ...- on simple and other complex data types (just like EOs are constructed from information elements and EOs). They represent the objects (physical and informational) which are manipulated in the enterprise.

ALBERT does not provide any explicit representation of object views. An object view would correspond in ALBERT to a particular data type which is a sub-sort of another. For example, using this mechanism makes possible to define a cartesian product of three components as a subset of a cartesian product of five components.

ALBERT provides usual abstraction mechanisms associated with abstract data types (viz genericity and specialisation) and thereby can be used for the definition of generic objects (encompassing several IEs or EOs) and the definition of specialisation of them.

Objects Relationships can be expressed by two means: cartesian products or tables. Both solutions can equally be used. The choice of a solution rather than the other depends on constraints and accesses that have to be expressed on the relation. The expression may be easier with a table in some cases, and more easier with a Cartesian product in others.

Integrity rules are easily expressed in ALBERT where, under the state behaviour heading (see Sect. 2.3), any temporal logic formula can be used. This enables the expression of constraints expressing derivation of values of components from values of others, invariants and constraints on the evolution of states.

\subsection{The Design Model}

In CIMOSA, the Design Specification Modelling Level is concerned with the technological choices to be made for the implementation of the CIM system. At that level, the major design decision is concerned with the identification of the actual resources (called Functional Entities in CIMOSA) that will be able to carry out the different elementary functions identified during the Requirements Definition level. In other words, at the design level, we need to transform the idealized (process-driven) view of the manufacturing system into a degraded view of the system where constraints are superimposed due to the nature and the capabilities of the different resources which are used.

In CIMOSA, the resulting design model presents the different functional entities together with the behaviour of the activities that they perform. In ALBERT, the hierarchical structure of the resources is reflected in the graphical diagram presented in Figure 4 where the agents correspond to the different functional entities. In the particular case of the CementBin resource, we have presented its complete specification in Figure 3. 


\title{
Cement Weight
}

\author{
BASIC CONSTRAINTS \\ INITIAL VALUATION \\ CementStore $=0 \wedge$ RequiredQty $=0 \wedge$ WeighedQuantity $=0$

\section{LOCAL CONSTRAINTS} \\ EFFECTS OF ACTIONS

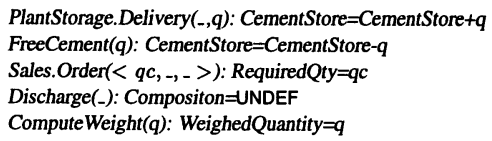

\section{CAusality}

Sales.Order $\left(<q c,,_{-,},>\right) \stackrel{\diamond}{\longrightarrow}$ Exception $\oplus((\text { FreeCement(-) }))^{*}$; Exception $) \oplus\left((\text { FreeCement(-) })^{*} ;\right.$ Discharge $\left.(q c)\right)$ Exceptions may occur during cement weighing: either at the beginning or after a certain quantity (below the required quantity) of cement has been weighed.

CAPABILITY

$\mathcal{X O}$ ( ComputeWeight $(q) /$ RequiredQty $\neq 0 \wedge$ WeighedQuantity $<$ RequiredQty $)$ Weight computing occurs continiously from the order receipt until the required quantity has been weighed.

$\mathcal{F}$ ( FreeCement $(q)$ / WeighedQuantity $\geq$ RequiredQty $\vee q>$ CementStore )

No more cement than what is required, nor than what is available, can be weighed.

\section{COOPERATION CONSTRAINTS}

ACTION PERCEPTION

$\mathcal{X O}$ ( Sales.Order $\left(<q c,_{-,},>\right) /$CementStore $\geq q c \wedge$ RequiredQty $=0$ )

Sales orders are taken into account if sufficient stock exists for producing them and if no other order is already in production.

$\mathcal{X O}$ ( PlantStorage.Delivery(-,-)/TRUE )

Deliveries are always possible (capacities are infinite!)

ACTION INFORMATION

$\mathcal{O}$ ( Exception.ExceptionManagement / TRUE )

Exception Management is always informed of occurences of exceptions.

$\mathcal{O}$ ( Discharge(-).CementCarrying / TRUE )

Cement Carrying is always informed that the cement weighing activity is over.

Figure 10 ALBERT constraints associated with the CementWeight EA.

\section{CONCLUSION}

In this paper, we have used the ALBERT language, a formal requirements for the specification of real-time composite systems, in the context of CIM infrastructures modelling. In order to eval- 
uate the degree of expressiveness offered by ALBERT in this context, through the handling of a case study, we have compared it with respect to the modelling constructs proposed in CIMOSA for the requirements and design levels. From that experiment, we can draw two conclusions:

- The ALBERT language offers enough expressivity for capturing the different informal and semi-formal descriptions provided in the CIMOSA models;

- Thanks to the underlying formal semantics, ALBERT helps the analysts in detecting desired properties like e.g., the absence of deadlocks, the completeness of the specification, ....

Finally, we would like to conclude this paper by providing some insights about our researches in the CIM context. These researches are going in two directions:

1. On the one hand, we are working on the specification of CIM requirements fragments for the purpose of defining a library of reusable components for CIM applications. Such reusable components can be composed through the use of well-defined structuring mechanisms available in ALBERT but not fully detailed in this paper. Preliminary result is the identification and the specification of a general CIM framework presented in (Dubois, 1993).

2. On the other hand, we are working on the elaboration of a general architecture in terms of which functional as well non functional requirements can be reconciled within a unique formal requirements document. In CIMOSA, these non functional requirements are related, for example, to the Objectives and Constraints attached to Domains. Preliminary results are reported in (Dubois, 1994a).

\section{Acknowledgements}

Part of this work was supported by the Belgian Walloon Region under Project 2337 of the FIRST program and by the European Community under Project 8319 (MODELAGE) of the ESPRIT program.

\section{REFERENCES}

ESPRIT Consortium AMICE, editor. (1993) CIMOSA: Open System Architecture for CIM, volume 1 of Research Reports ESPRIT, Project 688/5288 AMICE. Springer-Verlag, 2nd revised and extended edition.

R. Bastide and C. Sibertin-Blanc. (1991) Modelling a flexible manufacturing system by means of cooperating objects. In G. Doumeingts, J. Browne, and M. Tomljanovich, editors, Computer Applications in Production and Engineering CAPE'91, pages 593-600, Bordeaux, September 10-12. IFIP, Elsevier Science Publishers B.V.

J. Brunet. (1991) Modelling the world with semantic objects. In Proc. of the working conference on the object-oriented approach in information systems, Québec (Canada).

Christoph Bussler. (1993) Enterprise process integration model and infrastructure. In H. Yoshikawa and J. Goosenaerts, editors, Preprints of JSPE-IFIP WG 5.3 Workshop on the Design of Information Infrastructure Systems for Manufacturing - DIISM'93, pages 415-426, Tokyo (Japan), November 8-10. Japan Society for Precision Engineering.

CAM.I. (1980) Architect's manual: ICAM definition method IDEF0. Technical Report DR-80ATPC-01, CAM.I., April. 
Eric Dubois, Philippe Du Bois, Frédéric Dubru, and Michaël Petit. (1994b) Agent-oriented requirements engineering: A case study using the albert language. In A. Verbraeck, H.G. Sol, and P.W.G. Bots, editors, Proc. of the Fourth International Working Conference on Dynamic Modelling and Information System - DYNMOD-IV, Noordwijkerhoud (The Netherlands), September 28-30. Delft University Press.

Eric Dubois, Philippe Du Bois, and Michaël Petit. (1993) Elicitating and formalising requirements for CIM information systems. In C. Rolland, F. Bodart, and C. Cauvet, editors, Proc. of the 5th conference on advanced information systems engineering - CAiSE'93, pages 252-274, Paris (France), June 8-11. LNCS 685, Springer-Verlag.

Eric Dubois, Jacques Hagelstein, and André Rifaut. (1991) A formal language for the requirements engineering of computer systems. In André Thayse, editor, From natural language processing to logic for expert systems, chapter 6 . Wiley.

Eric Dubois and Michael Petit. (1994a) The formal requirements engineering of manufacturing systems. In S.M. Deen, editor, Proc. of the Second International Working Conference on Cooperative Knowledge Based Systems - CKBS'94, pages 67-82, Keele (UK), June 14-17.

Martin S. Feather. (1987) Language support for the specification and development of composite systems. ACM Transactions on Programming Languages and Systems, 9(2):198-234, April.

Anthony Finkelstein and Colin Potts. (1987) Building formal specifications using "structured common sense". In Proc. of the 4th International Workshop on Software Specification and Design - IWSSD'87, pages 108-113, Monterey CA, April 3-4. IEEE, CS Press.

Sol J. Greenspan, Alexander Borgida, and John Mylopoulos. (1986) A requirements modelling language. Information Systems, 11(1):9-23.

Patrick Jaulent. (1990) Flexible manufacturing system specification with object-oriented method sys-p-otm. In Proc. of the International Conference CIM'90: Integration Aspects, pages 297306, Bordeaux (France), June 12-14. Productic-A, Teknea.

Eric Lutherer, Soumeya Ghroud, Michel Martinez, and Joël Favrel. (1994) Modelling with CIMOSA: A case study. In Proc. of the IFIP WG5.7 Working Conference on Evaluation of Production Management Methods, pages 233-241, Gramado (Brazil), March 21-24.

Douglas T. Ross. (1977) Structured analysis (sa): a language for communicating ideas. IEEE Transactions on software engineering, SE-3(1):16-34, January.

A. Sernadas, C. Sernadas, and H.-D. Ehrich. (1989) Abstract object types: a temporal perspective. In B. Banieqbal, H. Barringer, and A. Pnueli, editors, Proc. of the colloquium on temporal logic and specification, pages 324-350. LNCS 398, Springer-Verlag.

Mark S.Fox and Michael Gruninger. Ontologies for enterprise integration. In Michael Brodie, Mathias Jarke, and Michael Papazoglou, editors, Proc. of the Second International Conference on Cooperative Information Systems - CoopIS-94, pages 82-89, Toronto (Canada), May 1720, 1994.

Francois Vernadat. (1993) CIMOSA: Enterprise modelling and entegration using a process based approach. In H. Yoshikawa and J. Goosenaerts, editors, Preprints of JSPE-IFIP WG 5.3 Workshop on the Design of Information Infrastructure Systems for Manufacturing - DIISM'93, pages 161-175, Tokyo (Japan), November 8-10. Japan Society for Precision Engineering.

Noureddine Zerhouni and Hassane Alla. (1990) Continuous petri nets: a tool for production systems dynamic analysis. In Proc. of the International Conferefence CIM'90: Integration Aspects, pages 417-424, Bordeaux (France), June 12-14. Productic-A, Teknea. 